\title{
Quasilinear actions on products of spheres
}

\author{
Özgün Ünlü and Ergün Yalçın
}

\begin{abstract}
For some small values of $f$, we prove that if $G$ is a group having a complex (real) representation with fixity $f$, then it acts freely and smoothly on a product of $f+1$ spheres with trivial action on homology.
\end{abstract}

\section{Introduction}

Some of the most interesting questions about group actions on topological spaces are about group actions on products of spheres. Historically, these questions are generalizations of questions about group actions on a sphere. In general, one is interested in whether a given finite group $G$ can act freely on a product of $k$ spheres for a given integer $k \geqslant 1$. One of the long-lasting conjectures in this subject states that if a group $G$ acts freely on a product of $k$ spheres, then $\operatorname{rk}(G) \leqslant k$, where $\operatorname{rk}(G)$ is the rank of the group $G$ defined as the largest integer $s$ such that $(\mathbb{Z} / p)^{s} \subset G$ for some prime number $p$. In terms of construction of actions, it is known that every finite group can act freely on a product of spheres. However, the following problem is still open.

Problem. Show that every finite group can act freely on a product of spheres with trivial action on homology.

The type of action demanded in the above problem is much harder to construct since we are not allowed to permute the spheres in the product. In this paper, we are interested in the following question, which is closely related to the above problem.

Question. Suppose that $G$ is a finite group that has a complex representation or an oriented real representation with fixity $f$. Then, does it act freely and smoothly on a product of $f+1$ spheres with trivial action on homology?

Let $G$ be a finite group and $F$ be a field. The fixity of an $F$-representation $V$ of $G$ is defined as the maximum value of $\operatorname{dim}_{F} V^{g}$ among all $1 \neq g \in G$. Note that if $G$ has a complex representation with fixity $f$, then it acts freely on the coset space $U(n) / U(n-f-1)$, which has the integral homology of a product of $f+1$ spheres. So, the above problem is asking whether one can replace a free action on a space that has the homology of a product of spheres, with a free smooth action on a product of spheres.

It is clear that if $G$ has a representation with fixity zero, then it acts freely on a sphere. Adem, Davis, and Ünlü [1] showed that if $G$ has a complex representation $V$ of dimension $n$ with fixity 1 , then $G$ acts freely and smoothly on $S^{2 n-1} \times S^{4 n-5}$. They construct this action by

Received 13 May 2009; revised 14 April 2010; published online 5 October 2010.

2000 Mathematics Subject Classification 57S25 (primary), 20C15 (secondary).

The first author is partially supported by TÜBITAK-TBAG/109T384 and the second author is partially supported by TÜBA-GEBIP/2005-16. 
taking the unit sphere of the Whitney sum double of the tangent bundle of $S(V)$, where $S(V)$ denotes the unit sphere of $V$. We observe that their argument can be extended to some higher values of fixity. In particular, for complex representations we prove the following theorem.

THEOREM 1.1. If $G$ is a finite group that has a faithful $n$-dimensional complex representation with fixity 2 , then $G$ acts freely and smoothly on $X=S^{2 n-1} \times S^{4 n-5} \times S^{q(4 n-8)-1}$ for some $q \geqslant 2$ with trivial action on homology.

The value of $q$ in the above product depends on the value of $n$. For example, if $n \geqslant 4$, then the last sphere can be taken as low as $S^{8 n-17}$. This shows, in particular, that finite subgroups of $\mathrm{SU}(4)$ act freely and smoothly on $X=S^{7} \times S^{11} \times S^{15}$. We prove Theorem 1.1 using pullbacks of bundles over quaternionic Stiefel manifolds.

For real representations, we prove a similar result.

Theorem 1.2. Let $G$ be a finite group that has a faithful representation $\rho: G \rightarrow \operatorname{SO}(n)$ with fixity $f \leqslant 4$. Assume that $n \geqslant 12$ when $f=4$. Then $G$ acts freely and smoothly on a product of $f+1$ spheres with trivial action on homology.

For fixity $f \leqslant 3$, we can prove the above statement using a similar argument to the complex case, but for $f=4$ this method seems to fail. So we use a slightly different argument that involves a sequence of $\mathbb{R}$-algebras satisfying certain properties. The argument gives a recipe for constructing free actions for all values of $f$, but because of the well-known dimension restriction on division algebras over $\mathbb{R}$, at this point we can only make it work for $f \leqslant 4$.

Finally, we would like to remark that, as was explained in [1], to obtain a free action of a finite group on a finite complex homotopy equivalent to a product of $k$ spheres, it is enough to construct an action on a product of $k-1$ spheres with rank 1 isotropy. Then, using a technique given in [2], one gets a free action on a finite complex having homotopy type of a product of $k$ spheres. This allows us to state the following as a corollary of Theorems 1.1 and 1.2.

Corollary 1.3. Let $G$ be a finite group. If $G$ has a complex representation with fixity 3 , then $G$ acts freely on a finite complex $X$ homotopy equivalent to a product of four spheres with trivial action on homology. If $G$ has a real representation with fixity 5 having dimension $n \geqslant 12$, then $G$ acts freely on a finite complex $X$ homotopy equivalent to a product of six spheres with trivial action on homology.

We organize the paper as follows. In Section 2, some basic lemmas and Theorem 1.1 are proved. In this section, we also discuss how Theorem 1.2 can be proved for $f \leqslant 3$ using an argument similar to the complex case. In Section 3, we prove Theorem 1.2 for $f \leqslant 4$ using a more general argument.

\section{Proof of Theorem 1.1}

Throughout this section, we use the notation and terminology used in [6]. Let $F$ denote the field of real numbers $\mathbb{R}$, complex numbers $\mathbb{C}$, or quaternions $\mathbb{H}$. For a real number the conjugation is defined by $\bar{x}=x$, for a complex number $x=a+i b$ by $\bar{x}=a-i b$, and for a quaternion $x=a+i b+j c+k d$ by $\bar{x}=a-i b-j c-k d$. On the vector space $F^{n}$, we can define an inner 
product $(v, w)$ by taking

$$
(v, w)=v_{1} \bar{w}_{1}+v_{2} \bar{w}_{2}+\ldots+v_{n} \bar{w}_{n} .
$$

The classical group $U_{F}(n)$ is defined as the subgroup of $\operatorname{GL}(n, F)$ that preserves the inner product. For $F=\mathbb{R}, \mathbb{C}$, and $\mathbb{H}$, we have different notation for the group $U_{F}(n)$. The orthogonal group $O(n)$ is the subgroup of $\mathrm{GL}(n, \mathbb{R})$ formed by $n \times n$ real matrices $A$ satisfying the property $(A v, A w)=(v, w)$ for all $v, w \in \mathbb{R}^{n}$. Similarly, the unitary group $U(n)$ is the subgroup of $\mathrm{GL}(n, \mathbb{C})$ that preserves the inner product, and $\operatorname{Sp}(n)$ is the subgroup of $\mathrm{GL}(n, \mathbb{H})$ that preserves the inner product for vector space $\mathbb{H}^{n}$. We define classical groups $\mathrm{SO}(n)$ and $\mathrm{SU}(n)$ as subgroups of $O(n)$ and $U(n)$, respectively, formed by matrices with determinant equal to 1 .

In our proofs, we shall be using fibre bundles arising from Stiefel manifolds. Let $V_{k}\left(F^{n}\right)$ denote the subspace of $F^{n k}$ formed by the $k$-tuples of vectors $\left(v_{1}, v_{2}, \ldots, v_{k}\right)$ such that $v_{i} \in F^{n}$ for all $i=1, \ldots, k$, and for every pair $(i, j)$, we have $\left(v_{i}, v_{j}\right)=1$ if $i=j$ and zero otherwise. There is a homeomorphism between $V_{k}\left(F^{n}\right)$ and the coset space $U_{F}(n) / U_{F}(n-k)$ (see $[\mathbf{6}$, Theorem 1.3, Chapter 8]).

There is a sequence of fibre bundles

$$
V_{n}\left(F^{n}\right) \longrightarrow \cdots \longrightarrow V_{k+1}\left(F^{n}\right) \longrightarrow V_{k}\left(F^{n}\right) \longrightarrow \cdots \longrightarrow V_{2}\left(F^{n}\right) \longrightarrow V_{1}\left(F^{n}\right),
$$

where the map $q_{k}: V_{k+1}\left(F^{n}\right) \rightarrow V_{k}\left(F^{n}\right)$ is defined by $q_{k}\left(v_{1}, \ldots, v_{k+1}\right)=\left(v_{1}, \ldots, v_{k}\right)$ and the fibre of $q_{k}$ is $V_{1}\left(F^{n-k}\right)=S^{c(n-k)-1}$, where $c=\operatorname{dim}_{\mathbb{R}} F$ (see [6, Theorem 3.8, Corollary 3.9, Chapter 8]). Note that the sphere bundle $q_{k}: V_{k+1}\left(F^{n}\right) \rightarrow V_{k}\left(F^{n}\right)$ is the sphere bundle of the vector bundle $\bar{q}_{k}: \bar{V}_{k+1}\left(F^{n}\right) \rightarrow V_{k}\left(F^{n}\right)$, where $\bar{V}_{k+1}\left(F^{n}\right)$ is the space formed by $(k+1)$-tuples $\left(v_{1}, \ldots, v_{k+1}\right)$, where $\left(v_{1}, \ldots, v_{k}\right) \in V_{k}\left(F^{n}\right)$ and $\left(v_{i}, v_{k+1}\right)=0$ for all $i=1, \ldots, k$.

Lemma 2.1. The vector bundle $\bar{q}_{k}: \bar{V}_{k+1}\left(F^{n}\right) \rightarrow V_{k}\left(F^{n}\right)$ is stably trivial.

Proof. Note that a vector bundle $\xi$ is stably trivial if there is a bundle isomorphism $\xi \oplus \tau^{i} \cong$ $\tau^{j}$ for some trivial bundles $\tau^{i}$ and $\tau^{j}$ of dimensions $i$ and $j$, respectively. Note that in our case, we can consider the bundle $\xi=\left(\bar{q}_{k}: \bar{V}_{k+1}\left(F^{n}\right) \rightarrow V_{k}\left(F^{n}\right)\right)$ as a subbundle of the trivial bundle $V_{k}\left(F^{n}\right) \times F^{n}$ which is orthogonal to the bundle $\theta$ defined as follows. Let $\theta: E \rightarrow V_{k}\left(F^{n}\right)$ denote the bundle with total space

$$
E=\left\{\left(\left(v_{1}, v_{2}, \ldots, v_{k}\right), w\right) \mid w \in\left\langle v_{1}, \ldots, v_{k}\right\rangle,\left(v_{1}, \ldots, v_{k}\right) \in V_{k}\left(F^{n}\right), w \in F^{n}\right\}
$$

and with obvious projection map. It is easy to see that $\xi \oplus \theta$ is isomorphic to the trivial bundle $V_{k}\left(F^{n}\right) \times F^{n}$ and that $\theta$ is a trivial bundle.

Another way to see this result is the following. Note that a vector bundle $\xi$ over a paracompact base space $B$ is stably trivial if its classifying map $\hat{\xi}: B \rightarrow B U_{F}(n)$ becomes null-homotopic when it is composed with $B U_{F}(n) \rightarrow B U_{F}(n+i)$ for some $i$. In the above lemma, the classifying map for $\xi=\left(\bar{q}_{k}: \bar{V}_{k+1}\left(F^{n}\right) \rightarrow V_{k}\left(F^{n}\right)\right)$ is a map of the form $\phi: V_{k}\left(F^{n}\right) \rightarrow B U_{F}(n-k)$. Note that $B U_{F}(n-k)$ can be described as the space of $(n-k)$-dimensional subspaces in $F^{\infty}$. Under this description, the classifying map $\phi$ takes a $k$-tuple $\left(v_{1}, \ldots, v_{k}\right)$ of vectors in $F^{n}$ to the subspace orthogonal to the subspace generated by the vectors $v_{i}$.

We also have a fibration of the form

$$
V_{k}\left(F^{n}\right) \longrightarrow B U_{F}(n-k) \longrightarrow B U_{F}(n),
$$

which comes from the homeomorphism $V_{k}\left(F^{n}\right) \cong U_{F}(n) / U_{F}(n-k)$. The projection map $B U_{F}(n-k) \rightarrow B U_{F}(n)$ is given by inclusion of an $(n-k)$-dimensional subspace in $F^{\infty}$ to an $n$-dimensional subspace in $F^{\infty}$. The inclusion of a fibre over a subspace of dimension $n$ is exactly the map described above. Thus, the map $V_{k}\left(F^{n}\right) \rightarrow B U_{F}(n-k)$ in the above fibration coincides 
with the classifying map $\phi$. So, composing the classifying map $\phi$ with $B U_{F}(n-k) \rightarrow B U_{F}(n)$ gives a null-homotopic map. This shows that the bundle $\xi=\left(\bar{q}_{k}: \bar{V}_{k+1}\left(F^{n}\right) \rightarrow V_{k}\left(F^{n}\right)\right)$ is stably trivial.

Another important ingredient in our proofs is a theorem about stably trivial bundles. Let $\xi$ be a vector bundle $E \rightarrow B$ over an $n$-dimensional $C W$-complex $B$. The following is a special case of a theorem given in $[\mathbf{6}]$.

Lemma $2.2[\mathbf{6}$, Theorem 1.5, Chapter 9]. Let $\xi$ be a $k$-dimensional $F$ vector bundle with $\operatorname{dim}_{\mathbb{R}} F=c$. Suppose that the base space $B$ is an $n$-dimensional CW-complex. If $\xi$ is stably trivial and $n \leqslant c(k+1)-2$, then $\xi$ is trivial.

Proof. Let $f: B \rightarrow B U_{F}(k)$ denote the classifying map for $\xi$. Assume that the composition of $f$ with $B U_{F}(k) \rightarrow B U_{F}(k+j)$ is null-homotopic for some $j$. Then $f$ lifts to a map $f$ : $B \rightarrow V_{j}\left(F^{k+j}\right)$. Using the fibration sequence for Stiefel manifolds, one can easily show that $\pi_{i}\left(V_{j}\left(F^{k+j}\right)\right)=0$ for $i \leqslant c(k+1)-2$. So, the result follows from the so-called compression lemma in homotopy theory (see [4, Lemma 4.6] or [3, Corollary 7.13]).

Lemma 2.2, in particular, shows that given a stably trivial vector bundle over a finitedimensional complex, we can obtain a trivial vector bundle by taking Whitney sum multiples of it sufficiently many times.

REMARK 2.3. Note that in vector bundle theory if the vector bundle in question is a smooth vector bundle $E \rightarrow B$ over a smooth manifold $B$, then a continuous trivialization can be replaced by a smooth trivialization leading to a diffeomorphism $S(E) \approx B \times S(V)$, where $S(E)$ is the total space of the corresponding sphere bundle. This is explained in detail in $[\mathbf{5}$, Chapter 4] (see also [7, Proposition 6.20]). Throughout the paper, we use these diffeomorphisms without further explanation. Note that the differential structure on the product $B \times S(V)$ is the product differential structure and $S(V)$ always denotes the standard sphere, not an exotic one.

Now we are ready to prove Theorem 1.1.

Proof of Theorem 1.1. Since the theorem obviously holds for the trivial group, we can assume $G \neq 1$. Let $V$ be a faithful complex representation of $G$ with $\operatorname{dim} V=n$ and fixity 2 . Since $V$ is faithful, we must have $n \geqslant 3$. Consider the pullback diagram

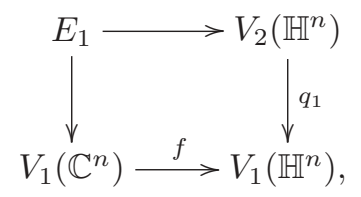

where $f$ is the map induced from the inclusion of $\mathbb{C}$ into $\mathbb{H}$ defined by $a+i b \rightarrow a+i b+$ $j 0+k 0$. Since the map $f: V_{1}\left(\mathbb{C}^{n}\right)=S^{2 n-1} \rightarrow V_{1}\left(\mathbb{H}^{n}\right)=S^{4 n-1}$ is null-homotopic, the bundle $E_{1} \rightarrow V_{1}\left(\mathbb{C}^{n}\right)$ is a trivial bundle with fibre $S^{4 n-5}$. So $E_{1} \approx S^{2 n-1} \times S^{4 n-5}$.

Now, consider the pullback bundle

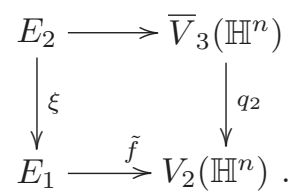


The bundle $\xi$ is stably trivial since it is a pullback of a stably trivial bundle. Taking a $q$-fold Whitney sum $\xi \oplus \ldots \oplus \xi$ of $\xi$, we obtain a bundle $\xi^{\oplus q}: E_{2}(q) \rightarrow E_{1}$. By Lemma 2.2, the bundle $\xi^{\oplus q}$ is a trivial bundle when

$$
\operatorname{dim} E_{1} \leqslant 4(q(n-2)+1)-2=4 q(n-2)+2 .
$$

Note that since $n \geqslant 3$, we can always find a $q$ that makes this inequality hold. In fact, since $\operatorname{dim} E_{1}=6 n-6$, the inequality holds even for $q=2$ when $n \geqslant 4$.

Note that the total space $E_{2}(q)$ can be considered as the subspace of $\mathbb{C}^{n(3+2 q)}$ formed by $(3+2 q)$-tuples of complex vectors $\left(v_{1,1}, v_{2,1}, v_{2,2}, v_{3,1}, v_{3,2}, \ldots, v_{3,2 q}\right)$ satisfying the property

$$
\left(\left(v_{1,1}, 0\right),\left(v_{2,1}, v_{2,2}\right),\left(v_{3,2 i-1}, v_{3,2 i}\right)\right) \in \bar{V}_{3}\left(\mathbb{H}^{n}\right)
$$

for all $i=1, \ldots, q$. Here, we consider a pair of complex numbers $(a+i b, c+i d)$ in $\mathbb{H}$ as the quaternion $y=a+i b+j c+k d$.

Let $V$ be an $n$-dimensional faithful complex representation of $G$, and let $W=\mathbb{H} \otimes_{\mathbb{C}} V$. By taking the average, we can assume that the inner product on $W$ is $G$-invariant. Note that if we consider elements of $W$ as pairs of complex vectors $\left(v_{1}, v_{2}\right)$, then the $G$-action on $W$ can be written as a diagonal action $g\left(v_{1}, v_{2}\right)=\left(g v_{1}, g v_{2}\right)$. From this it is easy to see that the $G$-action on $\bar{V}_{3}(W)$ gives a $G$-action on $E_{2}(q)$ for any $q$.

Let $X$ denote the total space of the sphere bundle of $E_{2}(q)$. Then $X \approx S^{2 n-1} \times S^{4 n-5} \times$ $S^{q(4 n-8)-1}$ and $G$ acts smoothly on $X$. Since $V$ has (complex) fixity 2 , the quaternionic representation $W$ has (quaternionic) fixity 2. This implies that if $g \in G$ fixes a point

$$
\left(v_{1,1}, v_{2,1}, v_{2,2}, v_{3,1}, v_{3,2}, \ldots, v_{3,2 q}\right) \in X,
$$

then we must have $v_{3, i}=0$ for all $i$, which is not possible. Thus, $G$ acts freely on $X$.

For real representations the argument given in the above proof can be extended to prove that if a finite group has a faithful real representation $\rho: G \rightarrow \mathrm{SO}(n)$ with fixity $f \leqslant 3$, then $G$ acts freely and smoothly on a product of $f+1$ spheres with trivial action on homology. For this, we consider the diagram

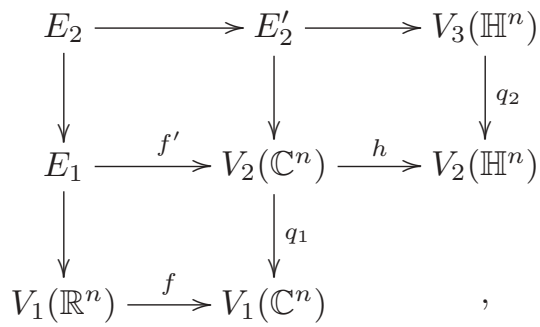

where each square in the diagram is a pullback square and the maps $f$ and $h$ are maps induced by the inclusions $\mathbb{R} \subset \mathbb{C} \subset \mathbb{H}$. Since the map $f$ is null-homotopic, it follows that $E_{1} \approx S^{n-1} \times$ $S^{2 n-3}$. The space $V_{2}(\mathbb{H})$ is $(4 n-6)$-connected. For $n \geqslant 2$, the inequality $\operatorname{dim} E_{1}=3 n-4 \leqslant$ $4 n-6$ holds, so by the compression lemma in homotopy theory, the composition $h \circ f^{\prime}$ is also null-homotopic. This implies that $E_{2} \approx S^{n-1} \times S^{2 n-3} \times S^{4 n-9}$.

Note that if $G$ has a real representation with fixity $f=1$ or 2 , then $G$ acts freely and smoothly on $E_{f}$ in the diagram. The $G$-actions are induced from the $G$-actions on Stiefel manifolds as in the complex case. For $f=3$, we first consider the pullback square

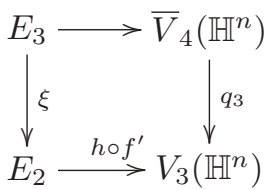


and then we take a $q$-fold Whitney sum of $\xi$ to make the dimension condition in Lemma 2.2 hold. For this we need $q$ to satisfy $\operatorname{dim} E_{2}=7 n-13 \leqslant q(4 n-12)+2$. Note that since we have $n \geqslant 4$, there always exists a $q$ that makes this inequality hold. If $n \geqslant 9$, the inequality holds even for $q=2$. Let $X$ denote the total space of the sphere bundle of $E_{3}(q)$; then $X$ is a product of four spheres. If $G$ has a faithful real representation $\rho: G \rightarrow \operatorname{SO}(n)$ with fixity $f=3$, then $G$ acts freely and smoothly on $X$.

Note that in the above construction the space $E_{1}$ can be considered as the space of tuples of $n$-dimensional real vectors $\left(v_{1,1}, v_{2,1}, v_{2,2}\right)$ such that $v_{1,1}+i 0$ is orthogonal to $v_{2,1}+i v_{2,2}$ as complex vectors. This is equivalent to saying that $v_{1,1} \perp v_{2,1}$ and $v_{1,1} \perp v_{2,2}$ as real vectors. It is clear from this that if $G$ acts orthogonally on $\mathbb{R}^{n}$, then it will act on $E_{1}$ with diagonal action on the coordinates. In a similar way, the space $E_{2}$ can be considered as the space of tuples of the form

$$
\left(v_{1,1}, v_{2,1}, v_{2,2}, v_{3,1}, v_{3,2}, v_{3,3}, v_{3,4}\right),
$$

where $v_{1,1} \perp v_{k, l}$ for all $k$ and $l$ with $k \geqslant 2$, and $v_{2,1}+i v_{2,2}$ is perpendicular to $v_{3,1}+i v_{3,2}$ and $v_{3,3}+i v_{3,4}$ as complex vectors. This is equivalent to saying that as pairs of real vectors, $\left(v_{2,1}, v_{2,2}\right)$ is perpendicular to $\left(v_{3,1}, v_{3,2}\right)$ and $\left(v_{3,3}, v_{3,4}\right)$, and that $\left(-v_{2,2}, v_{2,1}\right)$ is perpendicular to $\left(v_{3,1}, v_{3,2}\right)$ and $\left(v_{3,3}, v_{3,4}\right)$. Note that this second relation makes it possible to conclude that the $G$-action on $E_{2}$ is free for a representation with fixity $f=2$. If we had just taken all the tuples

$$
\left(v_{1,1}, v_{2,1}, v_{2,2}, v_{3,1}, v_{3,2}, v_{3,3}, v_{3,4}\right)
$$

with $v_{1,1} \perp v_{k, l}$ for all $k$ and $l$ with $k \geqslant 2$, and $\left(v_{2,1}, v_{2,2}\right)$ is perpendicular to $\left(v_{3,1}, v_{3,2}\right)$ and $\left(v_{3,3}, v_{3,4}\right)$, we would still have a product of spheres with a $G$-action but the action would no longer be free for a real representation with fixity 2 . In the next section, we elaborate on this idea and give a more general construction of 'quasilinear' actions.

Remark 2.4. In Theorem 1.2, if we start with an $O(n)$ representation instead of an $\mathrm{SO}(n)$ representation, then we still get a free action on a product of spheres, but the resulting action on homology will no longer be trivial since $G$ may act on some homology classes via the sign representation. However, the action on $\mathbb{F}_{2}$ homology will be trivial.

\section{Proof of Theorem 1.1}

Let $V$ be a real representation of $G$. Suppose that we are given a sequence of $\mathbb{R}$-algebras $A_{1} \subset A_{2} \subset \ldots \subset A_{s}$ which are possibly non-commutative and non-associative. For each $i$, define $V_{i}=V \otimes_{\mathbb{R}} A_{i}$. Then $V_{i}$ is a real representation with the $G$-action given by left multiplication $g(v \otimes a)=g v \otimes a$. Note that $V_{i}$ is also a module over $A_{i}$, where the action of $a^{\prime} \in A$ on $v \otimes a$ is given by $(v \otimes a) a^{\prime}=v \otimes\left(a a^{\prime}\right)$. In the non-associative case, this does not give a module structure in the usual sense but it still gives an $\mathbb{R}$-bilinear map $V_{i} \times A_{i} \rightarrow V_{i}$, which is what we need.

If there is an inner product on $V$, we can define an inner product on $V_{i}$ by $\left(v \otimes a, v^{\prime} \otimes\right.$ $\left.a^{\prime}\right)=\left(v, v^{\prime}\right)\left(a, a^{\prime}\right)$, where the inner products on the $\mathbb{R}$-algebras $A_{i}$ can be taken in such a way that $A_{i} \subset A_{i+1}$ is a subspace as an inner product space. This gives us a sequence of real representations of $G$

$$
V_{1} \subset V_{2} \subset \ldots \subset V_{s}
$$

such that each $V_{i}$ is a module over $A_{i}$.

From now on we assume that $V_{1} \subset V_{2} \subset \ldots \subset V_{s}$ is a sequence of real representations such that each $V_{i}$ is a module over $A_{i}$, but not necessarily of the form $V_{i}=V \otimes_{\mathbb{R}} A_{i}$ for a fixed real 
representation $V$. We choose an inner product on each $V_{i}$ such that $V_{i} \subset V_{i+1}$ is an inclusion of inner product spaces. For each $u \in V_{i}$, let $\langle u\rangle_{A_{i}}$ denote the vector space $\left\{u a \mid a \in A_{i}\right\}$. We denote the norm of a vector by $|u|$. We define

$$
W_{k}\left(\left\{V_{i}\right\}_{i=1}^{s}\right)=\left\{\left(v_{1}, v_{2}, \ldots, v_{k}\right) \mid v_{i} \in V_{i} \text { with }\left|v_{i}\right|=1 \text {, and } v_{j} \perp\left\langle v_{i}\right\rangle_{A_{j}} \text { for all } i<j\right\} .
$$

To simplify notation, we write $W_{k}(V)$ for $W_{k}\left(\left\{V_{i}\right\}_{i=1}^{s}\right)$. The subspace $\bar{W}_{k}(V)$ is defined as the space of $k$-tuples as above, but we no longer require the last vector $v_{k}$ to be a unit vector.

We will prove that under certain conditions, the map $q_{k}: \bar{W}_{k+1}(V) \rightarrow W_{k}(V)$ defined by $q_{k}\left(v_{1}, \ldots, v_{k+1}\right)=\left(v_{1}, \ldots, v_{k}\right)$ is a projection map of a stably trivial vector bundle. To find these conditions, we consider some subbundles of the trivial bundle $W_{k}(V) \times V_{k+1} \rightarrow W_{k}(V)$, which are defined in the following way. For each $i \in\{1, \ldots, k\}$, let

$$
E_{i}=\left\{\left(\left(v_{1}, \ldots, v_{k}\right), w\right) \mid w \in\left\langle v_{i}\right\rangle_{A_{k+1}},\left(v_{1}, \ldots, v_{k}\right) \in W_{k}(V)\right\},
$$

and let $q_{k, i}: E_{i} \rightarrow W_{k}(V)$ be the obvious projection map $\left(\left(v_{1}, \ldots, v_{k}\right), w\right) \rightarrow\left(v_{1}, \ldots, v_{k}\right)$.

Note that, for each non-zero $u \in V_{i}$, the vector space $\langle u\rangle_{A_{k+1}}$ is isomorphic to $A_{k+1} / \operatorname{Ann}_{A_{k+1}}(u)$, where $\operatorname{Ann}_{A_{k+1}}(u)=\left\{a \in A_{k+1} \mid u a=0\right\}$. Suppose that $\operatorname{Ann}_{A_{k+1}}(u)$ is equal to a fixed subspace $B_{k+1, i} \subset A_{k+1}$ for every $u \in V_{i}$. Then we can choose a basis $\left\{a_{1}, \ldots, a_{m}\right\}$ for $A_{k+1}$ such that $\left\{a_{1}, \ldots, a_{l}\right\}$ is a basis for $B_{k+1, i}$. Using this basis, we can easily express $w \in\left\langle v_{i}\right\rangle_{A_{k+1}}$ uniquely as $\sum_{t=l+1}^{m} \gamma_{t}\left(v_{i} a_{t}\right)$, where $\gamma_{t} \in \mathbb{R}$. We conclude the following.

Lemma 3.1. Suppose that $\operatorname{Ann}_{A_{k+1}}(u)$ is equal to a fixed subspace $B_{k+1, i} \subset A_{k+1}$ for every non-zero $u \in V_{i}$. Then the map $q_{k, i}: E_{i} \rightarrow W_{k}(V)$ defined above is the projection map of a fibre bundle $\theta_{k, i}$ which is a trivial bundle with dimension $\operatorname{dim}_{\mathbb{R}}\left(A_{k+1} / B_{k+1, i}\right)$.

If a filtration $\left\{V_{i}\right\}_{i=1}^{s}$ satisfies the condition given in the above lemma for all $i$ and $k$ with $1 \leqslant i \leqslant k \leqslant s-1$, then we say it is a uniform filtration. In our applications, this condition holds often with $B_{k+1, i}=0$ for all $i, k$. Another condition that we can impose on our filtration is the following.

Definition 3.2. We say that a sequence $\left\{V_{i}\right\}_{i=1}^{s}$ is separable at $k$ if, for every $\left(v_{1}, \ldots, v_{k}\right) \in$ $W_{k}(V)$, we have

$$
\left\langle v_{j}\right\rangle_{A_{k+1}} \cap\left\langle v_{i}\right\rangle_{A_{k+1}}=0
$$

for every $i$ and $j$, with $1 \leqslant i<j \leqslant k$. Then we say that the sequence $\left\{V_{i}\right\}_{i=1}^{s}$ is a separable sequence if it is separable at every $k$, with $1 \leqslant k \leqslant s-1$.

For a uniform and separable sequence, of real representations, the following is true.

Lemma 3.3. Let $\left\{V_{i}\right\}_{i=1}^{s}$ be a uniform separable sequence. Then the space

$$
E=\left\{\left(\left(v_{1}, \ldots, v_{k}\right), w\right) \mid w \in \sum_{i=1}^{k}\left\langle v_{i}\right\rangle_{A_{k+1}},\left(v_{1}, \ldots, v_{k}\right) \in W_{k}(V)\right\},
$$

together with the obvious projection map $E \rightarrow W_{k}(V)$, defines a vector bundle isomorphic to $\theta_{k, 1} \oplus \theta_{k, 2} \oplus \ldots \oplus \theta_{k, k}$.

Let $\xi_{k}$ denote the orthogonal complement of the bundle $\theta_{k, 1} \oplus \ldots \oplus \theta_{k, k}$ in the trivial bundle $\tau_{k}: W_{k}(V) \times V_{k+1} \rightarrow W_{k}(V)$. Then it is not very difficult to see that $\xi_{k}$ is the bundle with the projection map $q_{k}: \bar{W}_{k+1}(V) \rightarrow W_{k}(V)$ described above. So, we conclude the following. 
Proposition 3.4. Let $\left\{V_{i}\right\}_{i=1}^{s}$ be a uniform separable sequence of real representations. Then the vector bundle $\xi_{k}: \bar{W}_{k+1}(V) \rightarrow W_{k}(V)$ is a stably trivial vector bundle of dimension $\operatorname{dim}_{\mathbb{R}} V_{k+1}-\sum_{i=1}^{k} \operatorname{dim}_{\mathbb{R}}\left(A_{k+1} / B_{k+1, i}\right)$.

Let $m_{1}=\operatorname{dim}_{\mathbb{R}} V_{1}$ and, for each $1 \leqslant k \leqslant s-1$, define

$$
m_{k+1}=\operatorname{dim}_{\mathbb{R}} V_{k+1}-\sum_{i=1}^{k} \operatorname{dim}_{\mathbb{R}}\left(A_{k+1} / B_{k+1, i}\right) .
$$

Note that a CW-structure can be given for the space $W_{k}(V)$, and as a CW-complex the dimension of $W_{k}(V)$ is $\sum_{i=1}^{k}\left(m_{i}-1\right)$. By Lemma 2.2, we have the following.

Proposition 3.5. Let $\left\{V_{i}\right\}_{i=1}^{s}$ be a uniform separable sequence of representations. Suppose that

$$
\sum_{j=1}^{k}\left(m_{j}-1\right) \leqslant m_{k+1}-1
$$

holds for all $1 \leqslant k \leqslant s-1$. Then the vector bundles $\xi_{1}, \ldots, \xi_{s}$ associated to this sequence are all trivial vector bundles. In particular, for each $k \leqslant s$ we have $W_{k}(V) \approx S^{m_{1}-1} \times S^{m_{2}-1} \times$ $\ldots \times S^{m_{k}-1}$.

Now we are ready to prove Theorem 1.2.

Proof of Theorem 1.2. Let $G$ be a finite group and $V$ be a faithful $n$-dimensional oriented real representation of $G$ with fixity 4 . We assume that $n \geqslant 12$. Consider the sequence of $\mathbb{R}$-algebras

$$
A_{1}=\mathbb{R} \subset A_{2}=\mathbb{C} \subset A_{3}=\mathbb{H} \subset A_{4}=\mathbb{O} \subset A_{5}=\mathbb{S},
$$

where $\mathbb{O}$ denotes the octonions and $\mathbb{S}$ denotes the sedenions. These algebras are the first five steps of the Cayley-Dickson construction, where $A_{i}$ is defined as the pairs of elements $(a, b)$ with $a, b \in A_{i-1}$ and the multiplication is given by

$$
(a, b)(c, d)=\left(a c-d^{*} b, d a+b c^{*}\right),
$$

and the conjugation for a pair is defined by $(a, b)^{*}=\left(a^{*},-b\right)$.

One particular property of the above sequence is that, for every $a_{i} \in A_{i}$ and $a_{j} \in A_{j}$, with $1 \leqslant i<j \leqslant 5$, if $a_{i} a_{j}=0$, then $a_{i}=0$ or $a_{j}=0$. This is because the algebras $\mathbb{R}, \mathbb{C}, \mathbb{H}$, and $\mathbb{O}$ are division algebras. The algebra $\mathbb{S}$ is not a division algebra, but we have $(a, 0)(c, d)=0 \Rightarrow$ $a c=0, d a=0$, so the above property still holds since $\mathbb{O}$ is a division algebra. Because of this property, we can conclude that the sequence $\left\{V_{i}\right\}_{i=1}^{5}$ with $V_{i}=V \otimes_{\mathbb{R}} A_{i}$ is a uniform sequence with $B_{k+1, i}=0$ for all $1 \leqslant i \leqslant k \leqslant 4$.

We claim that the sequence $\left\{V_{i}\right\}_{i=1}^{5}$ is also separable. To show this, we need to show that, for every $k \leqslant 4$, the following holds. If $v_{i} \in V_{i}$ and $v_{j} \in V_{j}$, with $1 \leqslant i<j \leqslant k$, then $\left\langle v_{i}\right\rangle_{A_{k+1}} \cap$ $\left\langle v_{j}\right\rangle_{A_{k+1}}=0$. Note that, for every $u, t$ with $1 \leqslant u<t \leqslant 5$, the algebra $A_{t}$ is a free module over $A_{u}$. So, to show that $\left\langle v_{i}\right\rangle_{A_{k+1}} \cap\left\langle v_{j}\right\rangle_{A_{k+1}}=0$, it is enough to show that $\left\langle v_{i}\right\rangle_{A_{j}} \cap\left\langle v_{j}\right\rangle_{A_{j}}=0$. By choosing a free basis for $A_{j}$ as an $A_{i}$-module, we can express $v_{i}$ in $V_{j}$ as a tuple $\left(v_{i}, 0, \ldots, 0\right)$ and $v_{j}$ as a tuple $\left(v_{j, 1}, \ldots, v_{j, d}\right)$, where $d=\operatorname{dim}_{A_{i}} A_{j}$.

If $\left\langle v_{i}\right\rangle_{A_{j}} \cap\left\langle v_{j}\right\rangle_{A_{j}} \neq 0$, then there exists an $a \in A_{i}$ such that $v_{i} a=\sum_{l=1}^{d} v_{j, l} b_{l}$ for some $b_{l} \in$ $A_{i}$. Since $i<4$, the algebra $A_{i}$ is an associative division algebra, so $b_{l}$ is invertible. Using the fact that $v_{j} \perp\left\langle v_{i}\right\rangle_{A_{j}}$, we obtain that $v_{i} a\left(b_{l}\right)^{-1} \perp v_{j, l}$ for all $l$. This gives that $v_{i} a$ is perpendicular 
to $v_{j, l} b_{l}$ for all $l$. But then $v_{i} a$ would be perpendicular to itself, which is a contradiction. So, the sequence $\left\{V_{i}\right\}_{i=1}^{5}$ is a separable sequence.

Since $B_{k+1, i}=0$ for all $k$ and $i$ with $1 \leqslant i \leqslant k \leqslant 4$, we have $m_{1}=\operatorname{dim}_{\mathbb{R}} V_{1}=n$ and

$$
m_{k+1}=\operatorname{dim}_{\mathbb{R}} V_{k+1}-\sum_{i=1}^{k} \operatorname{dim}_{\mathbb{R}}\left(A_{k+1} / B_{k+1, i}\right)=n 2^{k}-\sum_{i=1}^{k} 2^{k}=(n-k) 2^{k} .
$$

Thus, to apply Proposition 3.5, we need the following inequalities to hold:

$$
\begin{aligned}
n-1 & \leqslant 2 n-3, \\
3 n-4 & \leqslant 4 n-9, \\
7 n-13 & \leqslant 8 n-25, \\
15 n-38 & \leqslant 16 n-65 .
\end{aligned}
$$

Note that the first three inequalities are satisfied when $n \geqslant 12$. The last inequality needs a higher bound for $n$, but we can always use the Whitney sum on the last bundle, so we really do not need the last inequality to hold. To see this, consider the bundle $\bar{W}_{5}(V) \rightarrow W_{4}(V)$. Since the sequence $\left\{V_{i}\right\}_{i=1}^{4}$ is splitting, we get $W_{4}(V) \cong S^{n-1} \times S^{2 n-3} \times S^{4 n-9} \times S^{8 n-25}$. Taking a $q$-fold Whitney sum of this bundle, we get a real vector bundle, say $E(q)$, with dimension $q(16 n-64)-1$. For this bundle to be trivial, we need the inequality

$$
15 n-38 \leqslant q(16 n-64)-1
$$

to hold. Since we have already assumed $n \geqslant 12$, the inequality holds even for $q=2$.

Finally, we need to show that $G$ acts freely on the total space of the sphere bundle for $E(q)$. Let us denote this space by $X$. As before, we consider an element in $E(q)$ as a tuple of real vectors $\left(v_{1,1}, v_{2,1}, v_{2,2}, \ldots, v_{5,1}, \ldots, v_{5,16 q}\right)$ in $V$ which satisfies certain orthogonality conditions. Note that the inner product on $V_{5}$ respects the decomposition of it as a direct sum of smaller vector spaces $V_{i}$, so all these orthogonality conditions can be expressed in terms of these real coordinate vectors. If we choose the inner product on $V$ as a $G$-invariant inner product, then $G$ will act on $E(q)$ and hence on $X$. Thus, $G$ acts smoothly on $X$.

Finally, we will show that the action is free when the fixity $f$ of $V$ is 4 . Suppose that we have an element $\left(v_{1,1}, v_{2,1}, v_{2,2}, \ldots, v_{5,1}, \ldots, v_{5,16 q}\right)$ fixed by $1 \neq g \in G$. Then, the vector $\left(v_{5,1}, \ldots, v_{5,16}\right)$ will lie in $V_{5}^{g}$ and will be orthogonal to subspaces $\left\langle v_{i}\right\rangle_{A_{5}}$ of $V_{i}^{g}$ for every $v_{i} \in V_{i}$. Here $v_{1}=v_{1,1}, v_{2}=\left(v_{2,1}, v_{2,2}\right), v_{3}=\left(v_{3,1}, \ldots, v_{3,4}\right)$, and $v_{4}=\left(v_{4,1}, \ldots, v_{4,8}\right)$. So, $\left(v_{5,1}, \ldots, v_{5,16}\right)$ lies in a vector space of dimension $16 f-16 \cdot 4$ which is zero when $f=4$. So, $G$ acts freely on $X$.

We conclude the paper with the proof of Corollary 1.3.

Proof of Corollary 1.3. Let $V$ be a complex representation of $G$ with fixity $f=3$. Repeating the argument in Theorem 1.1, we obtain a smooth action of $G$ on

$$
X=S^{2 n-1} \times S^{4 n-5} \times S^{q(4 n-8)-1} .
$$

Note that if $H$ is a subgroup of $G$ that fixes a point on $X$, then it also fixes a point on $V_{3}(W)$, where $W=\mathbb{H} \otimes_{\mathbb{C}} V$. Since $V$ has fixity 3, it acts freely on $V_{4}(W)$. Thus, $H$ acts freely on the fibre of $q_{3}: V_{4}(W) \rightarrow V_{3}(W)$. The fibre of this map is homeomorphic to a sphere, so $H$ has periodic cohomology by classical Smith theory. Now, from [2, Theorem 3.2], we can conclude that $G$ acts freely on a finite complex $Y$ homotopy equivalent to $X \times S^{N}$ for some $N$.

In the real case the proof is similar. Let $X$ be the $G$-space constructed in the proof of Theorem 1.2. Now suppose that $H$ is a subgroup of $G$ fixing a point

$$
\left(v_{1,1}, v_{2,1}, v_{2,2}, \ldots, v_{5,1}, \ldots, v_{5,16 q}\right) \in X
$$


Then we can find indices $i_{j}$ such that the 5 -tuple $\left(v_{1,1}, v_{2, i_{2}}, v_{3, i_{3}}, v_{4, i_{4}}, v_{5, i_{5}}\right)$ is a point on the Stiefel manifold $V_{5}(V)$, and it is fixed by $H$ under the usual $G$ action on the Stiefel manifold. Since $G$ acts freely on $V_{6}(V)$, we obtain that $H$ acts freely on the fibre of $V_{6}(V) \rightarrow V_{5}(V)$. The rest follows as in the complex case.

Acknowledgement. We thank the referee for a careful reading of the paper and for many helpful comments.

\section{References}

1. A. Adem, J. F. DAVIS and Ö. ÜnLÜ, 'Fixity and free group actions on products of spheres', Comment. Math. Helv. 79 (2004) 758-778.

2. A. Adem and J. H. Smith, 'Periodic complexes and group actions', Ann. of Math. (2) 154 (2001) 407-435.

3. J. F. DAVIS and P. KIRK, Lecture notes in algebraic topology, Graduate Studies in Mathematics 35 (American Mathematical Society, Providence, RI, 2001).

4. A. Hatcher, Algebraic topology (Cambridge University Press, Cambridge, 2002).

5. M. W. Hirsch, Differential topology (Springer, New York, 1976).

6. D. Husemoller, Fibre bundles, 3rd edn, Graduate Texts in Mathematics 20 (Springer, New York, 1994).

7. J. M. LEE, Introduction to smooth manifolds, Graduate Texts in Mathematics 218 (Springer, New York, 2003).

Özgün Ünlü and Ergün Yalçın

Department of Mathematics

Bilkent University

Ankara 06800

Turkey

unluo@fen.bilkent.edu.tr

yalcine@fen.bilkent.edu.tr 